\title{
Study of Eddy Currents Non Destructive Testing System in Riveted Assemblies
}

\author{
Jean Louis Rasolonjanahary, Frédéric Thollon, Noël Burais \\ CEGELY - URA CNRS 829 - Ecole Centrale de Lyon - Département d'Electrotechnique \\ BP 163 - 69131 Ecully Cedex - France \\ Xavier Brunotte \\ Cedrat-Recherche - 10 chemin du Pré-Carré - 38240 Meylan - France
}

\begin{abstract}
In order to optimize eddy current sensors, we have to simulate electromagnetic phenomena to predict sensors' response. 2D and 3D codes can be used but what kind of result can be expected from each of these codes? In this paper, performances of 3D formulations are tested using FEM package Flux $3 d$. Adapted coupled formulations and boundary conditions are used to study eddy currents perturbation by flaws in aircraft riveted assemblies. Physical informations about the influence of flaw depth are obtained. The 3D calculations allow us to choose the most adapted measurement quantity and to define the position of the measurement sensors. Then, for optimizing the sensor, 2D FEM package Fissure is used taking into account the 3D results.
\end{abstract}

\section{INTRODUCTION}

Eddy currents are widely used for Non Destructive Testing of conducting structures. Practically, characteristics of the device as coils sizing or excitation frequency are chosen using results of simple models which predict approximate sensor performances. Generally, when the test concerns the area near the surface of the material, this knowledge of sensor parameters is sufficient to perform efficient measurements. For deeper testing, as inspection of flaws under installed fasteners in aircraft structures, this empirical method is not adapted. In order to understand electromagnetic phenomena in tested structures and to determine the sensor response according to various physical parameters, Maxwell's relations can be solved with 2D or 3D classical numerical methods. But several difficulties are related to this control method:

a) very small variation of sensor response when a flaw exists,

b) many geometrical and electrical parameters can modify this response,

c) eddy currents decrease rapidly in the tested material because of the skin effect.

Two complementary ways can be followed:

- 3D simulation which allows accurate results for various structures but implies time and CPU computer consuming. Difficulties are also introduced by the sizes of the flaw, particularly the width.
- 3D-axisymmetric (2D) simulation which gives fewer accurate results but allows parameters studies in order to more quickly design the sensor. This is possible on structures like riveted assemblies where rivet and coil are axisymmetric $[4,5]$.

The aim of the paper is to define the most adapted formulation and to study 3D eddy currents distribution near the flaw. 3D and 2D results about sensor response (flux density at the surface of material) are compared. It is demonstrated that the $2 \mathrm{D}$ simulation with an equivalent axisymmetric flaw can be used to design a performant sensor for these structures after a 3D study which allows to define the sensor structure (measurement quantity and flux density sensor position).

\section{GEOMETRICAL STRUCTURE OF CONTROLLED SYSTEM}

Typical riveted assembly in aircraft structure and excitation coil are presented on Fig. 1. All materials are non magnetic. The metallic sheets are highly conductive. In such a structure, flaws can occur along the rivet at important depth $(>5 \mathrm{~mm}$ ). Eddy currents distribution is modified by the flaw and can be detected by flux density variation (measurement with hall effect or magneto resistive sensor) at the surface of the sheet Fig. 2 shows the geometry used for the simulation.

\section{3D FORMULATIONS}

For simulating the response of a sensor in the presence of a defect, two kinds of finite elements methods exist: nodal and edge elements. For nodal finite elements method, there are two alternative formulations: $T-\Phi$ (electric vector potential and magnetic scalar potential) or $\mathrm{A}-\mathrm{V}$ (magnetic vector potential and electric scalar potential) [1]. Both are available in the finite element package Flux $3 D$ (distributed by CEDRAT). In order to get accurate results and to reduce the time computation, they must be coupled with others formulations and associated with wise boundary conditions. In this study, we use $\mathrm{A}-\mathrm{V}$ in conducting region $[2,3]$. In this case, the induced current density is:

$$
\mathbf{J}=-\sigma(j \omega \mathbf{A}+\nabla \mathrm{V})
$$




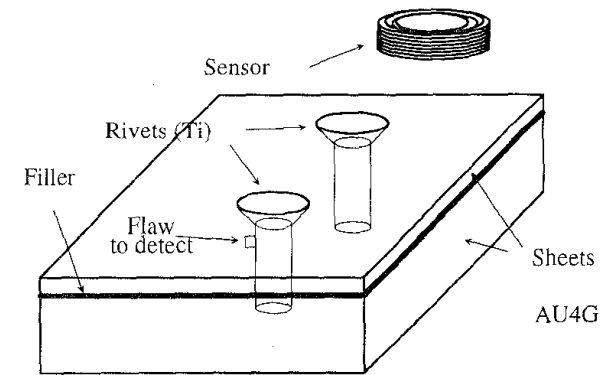

Fig. 1. Typical structure to control

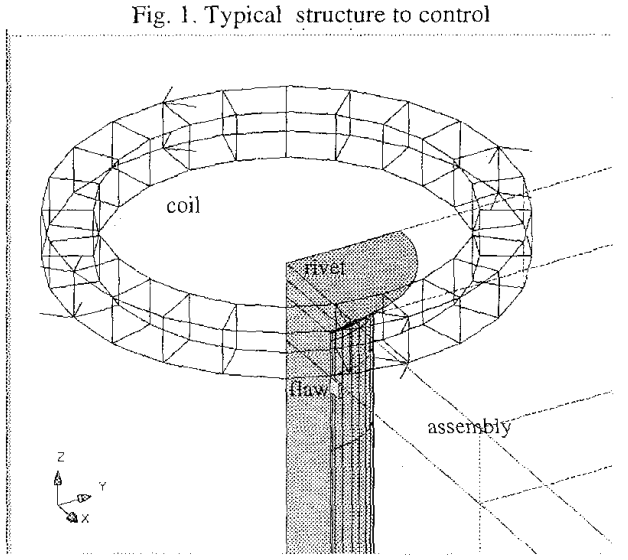

Fig. 2. Simulated structure

For the non-conducting region containing the sensor and the coil inductor, we use the reduced magnetic scalar potential $\Phi_{r}$. So the magnetic field measured by the sensor is given by:

$\mathbf{B}=\mu_{0}\left(\mathbf{H}_{\mathrm{s}}-\nabla \Phi_{\mathrm{r}}\right)$

with $\mathbf{H}_{\mathrm{s}}$ excitation field defined by:

$\nabla \times \mathbf{H}_{\mathrm{s}}=\mathbf{J}_{\mathrm{s}}$

Before we present the results relating to a deep flaw, we are interested in the influence of formulations used for the region occupied by the flaw. To do that, we use a surface flaw (easily detectable) with $0.25 \mathrm{~mm}$ width and $1 \mathrm{~mm}$ depth. The frequency of the calculation is $2000 \mathrm{~Hz}$. Three formulations can be used for the flaw, the magnetic vector potential $\mathbf{A}$, the total magnetic scalar potential $\Phi$ or the reduced magnetic scalar potential $\Phi_{\mathrm{r}}$. Five calculations were done using the sarne mesh:

1- without a defect,

2- $\mathbf{A}$ is used in the region occupied by the flaw without any condition at the interfaces flaw-conducting region,

3- $\mathbf{A}$ is used in the region occupied by the flaw and we cancel the normal component of $\mathbf{A}$ at these interfaces,

4- $\Phi$ is used in the region occupied by the flaw,

5- $\Phi_{\mathrm{r}}$ is used in the region occupied by the flaw.

For the two last cases, we cancel the normal component of $A$ at these interfaces in order to avoid numerical difficulties [2].

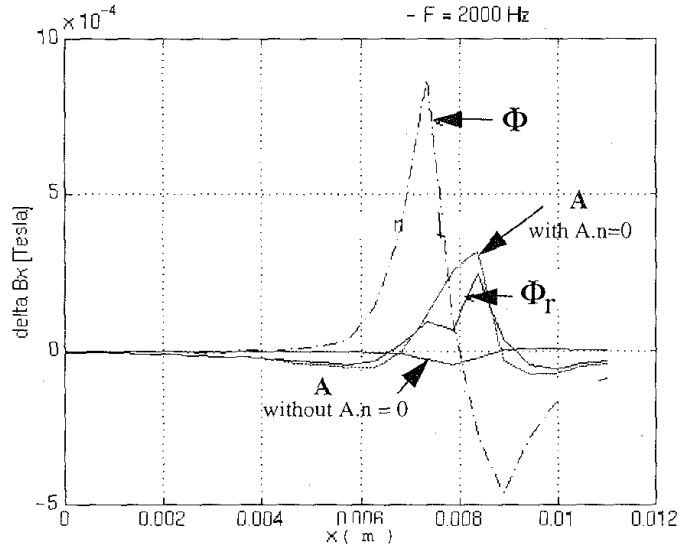

Fig. 3. Influence of formulations and conditions imposed on the flaw on the differential horizontal component of the flux density on the surface of the structure.

Among the results, we show on Fig. 3 the evolution of the differential flux density $\Delta \mathrm{B}_{\mathrm{x}}$ (horizontal component of flux density without a flaw - horizontal component of flux density with a flaw) according to a radial line located at midway between the inductor and the sheet.

In this figure, we observe the disparities due to the formulations used in the flaw and the influence of boundary conditions imposed on the interfaces.

For $\Phi$ and $\Phi_{r}$ formulations, the normal component $B_{n}$ is ensured in a weak sense at these interfaces. Using $\mathbf{A}$ in the flaw ensures the exact continuity of $B_{n}$. Moreover, at these interfaces, the normal component of current density is given by

$$
J_{n}=-\sigma\left(j \omega A_{n}+\frac{\partial V}{\partial n}\right)
$$

Since $\frac{\partial V}{\partial n}=0$ is a natural condition, the fact of imposing $A_{n}=$ zero gives us the exact continuity of $J_{n}$. Otherwise, if we did not so, the continuity of $J_{n}$ is ensured in a weak sense, it means, locally $J_{n}$ is not zero. So, in the flaw region, we use the magnetic vector potential $A$ with the condition $A_{n}=$ zero at the interfaces flaw-conducting region.

\section{RESULTS}

Previous formulations are used in finite element package Flux3D for studying eddy-current density distribution around various flaws at $5 \mathrm{~mm}$ depth (Fig. 4 and 5).

For each case, the frequency is equal to $500 \mathrm{~Hz}$. A computation of the perturbation is performed by calculating the current through surfaces near the flaw (Fig 6):

-) current in front of the flaw ( surface A).

-) current above the flaw ( surface C).

-) current below the flaw ( surface D).

Results about current distribution around the flaw are shown in Table 1. 


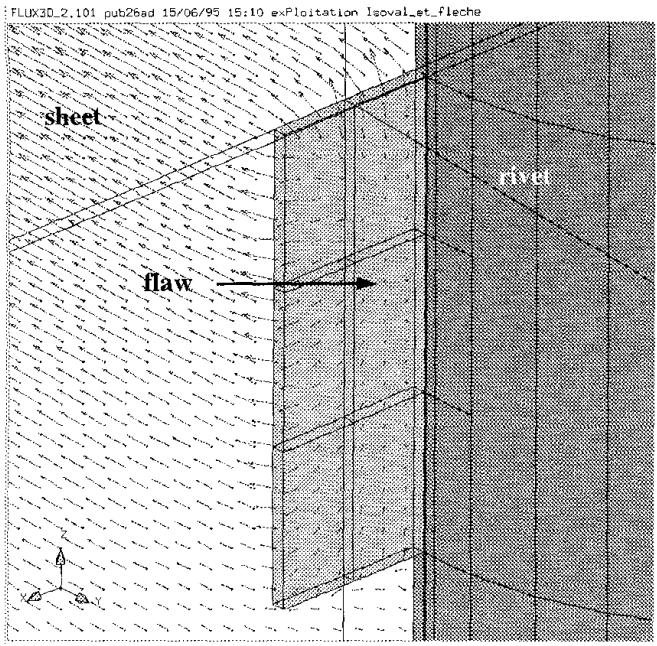

Fig. 4. Disturbance of current density for a $2 \mathrm{~mm} \times 6 \mathrm{~mm}$ flaw.

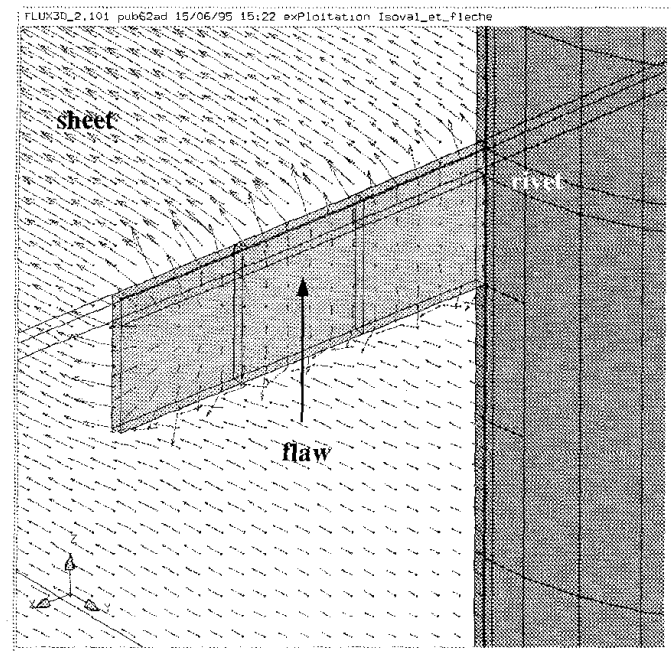

Fig. 5. Perturbation of current density for a $6 \mathrm{mmx} 2 \mathrm{~mm}$ flaw.

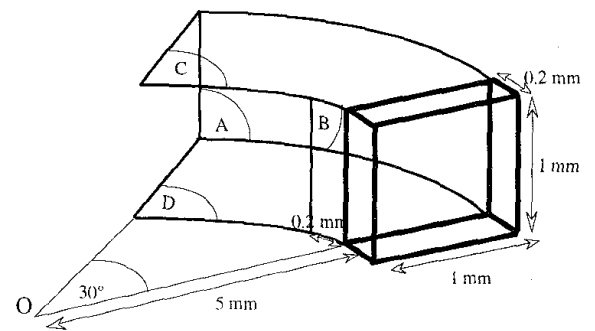

Fig. 6. Description of the flaw and surfaces used to calculate perturbation.

As expected, eddy currents are especially deviated in a horizontal plan for a vertical defect, while they are deviated in a vertical plan for of radial defects.

For this type of structure, the control is made by measuring the flux density on the surface of the material along a segment above the defect.
TABLE 1

Current Distribution Around The Flaw

\begin{tabular}{lccc}
\hline $\begin{array}{l}\text { flaw sizes } \\
\text { width/height }\end{array}$ & $\begin{array}{c}\text { above the } \\
\text { flaw }\end{array}$ & $\begin{array}{c}\text { below the } \\
\text { flaw }\end{array}$ & $\begin{array}{c}\text { in front of } \\
\text { the flaw }\end{array}$ \\
\hline $2 \mathrm{~mm} \times 2 \mathrm{~mm}$ & $26 \%$ & $36 \%$ & $38 \%$ \\
$2 \mathrm{~mm} \times 4 \mathrm{~mm}$ & $18 \%$ & $27 \%$ & $55 \%$ \\
$2 \mathrm{~mm} \times 6 \mathrm{~mm}$ & $11 \%$ & $24 \%$ & $\mathbf{6 5 \%}$ \\
$4 \mathrm{~mm} \times 2 \mathrm{~mm}$ & $38 \%$ & $36 \%$ & $26 \%$ \\
$6 \mathrm{~mm} \times 2 \mathrm{~mm}$ & $\mathbf{4 2 \%}$ & $39 \%$ & $19 \%$ \\
$4 \mathrm{~mm} \times 4 \mathrm{~mm}$ & $26 \%$ & $33 \%$ & $40 \%$ \\
\hline
\end{tabular}

The differential flux density is defined by the difference between the flux density without flaw and the flux density with a flaw. Fig. 7 and Fig. 8 give vertical and horizontal differential flux density for the same flaws as in table 1 .

As all calculations are undertaken with a constant frequency, only the radial dimension of the defect is significant. To differentiate defects following their height, it is necessary to work with several frequencies.

The most important conclusions concern the magnitude to

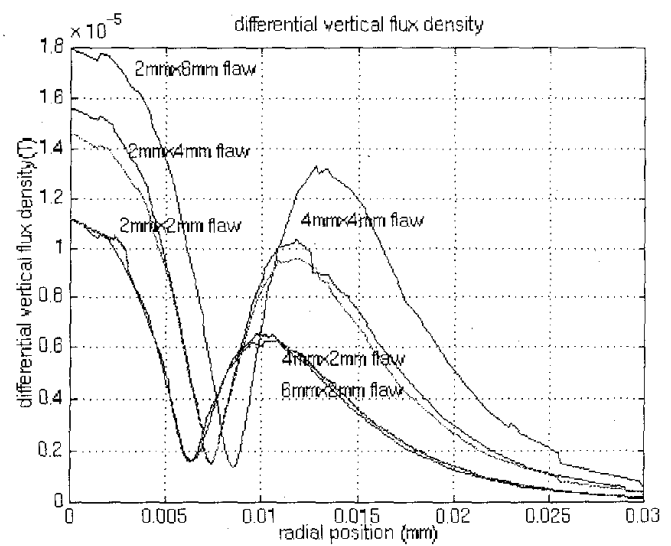

Fig. 7. Differential vertical flux density for different flaws.

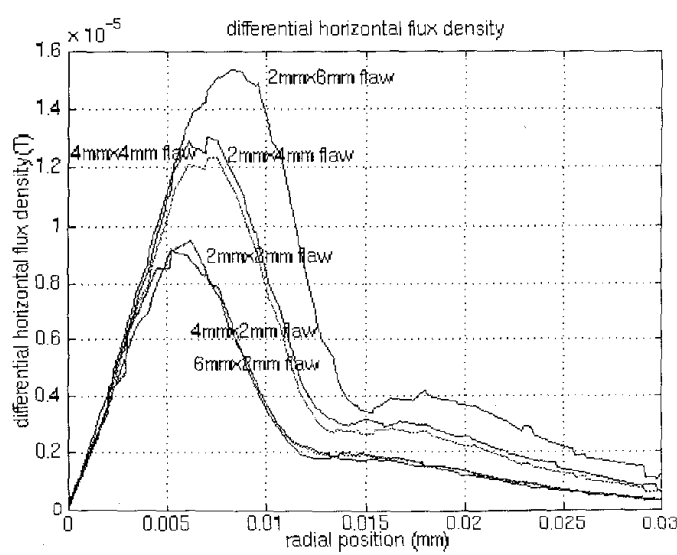

Fig. 8. Differential horizontal flux density for different flaws 
measure and the position of the corresponding sensor. Taking into account coil dimensions, we can see (Fig. 8) that the quantity to be considered is the horizontal component measured inside the coil (between 5 and $9 \mathrm{~mm}$ ).

Indeed, the variations of the vertical component don't allow a diagnosis because of the presence of a minimum whose position depends on the flaw sizes.

Moreover, Fig. 9 represents the horizontal differential flux density on the surface of the material. We can see the influence of the defect and thus define tolerances on the positioning of a flux density sensor.

Furthermore, the flux density is calculated at the surface of the sheet and compared with the result obtained with an axisymmetrical "flaw" (zero conductivity area) with the same cross-section as the 3D flaw. The calculation was performed with 3D and 2D packages (Fissure is a 2D code developed by CEGELY). We can see Fig. 10 that, for this controlled structure, "2D calculated" flux density variations are smaller. but in the same way that "3D calculated" flux density.

The design and the optimization of a sensor for the control of structures can be more quickly performed using 2D calculation associated with automatic optimization $[4,6]$.

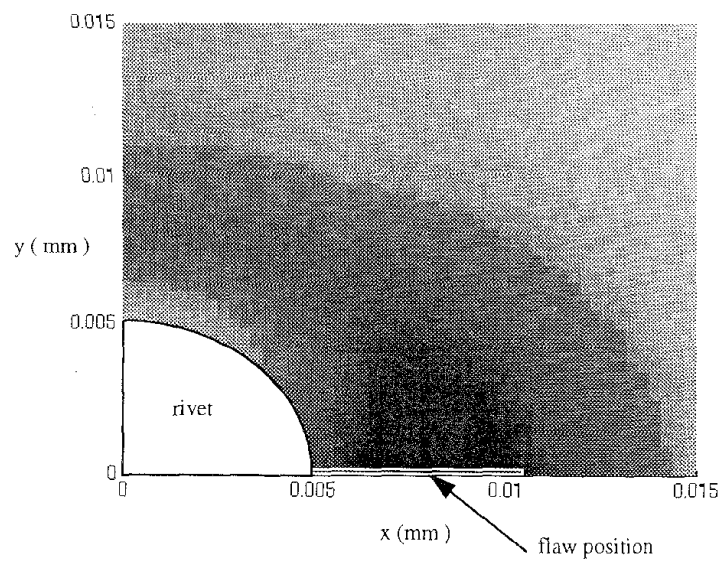

Fig. 9. Differential horizontal flux density above the surface for a $2 \mathrm{mmx} 6 \mathrm{~mm}$ flaw at $5 \mathrm{~mm}$ depth

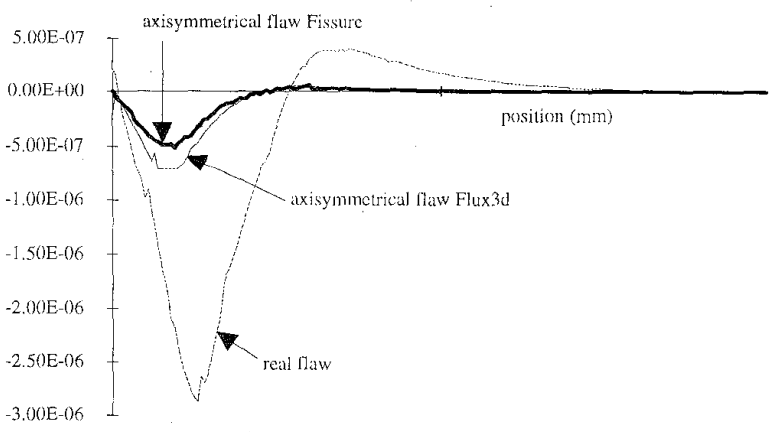

Fig. 10. Probe response for "real "flaw and axisymmetrical flaw (size $2 \mathrm{~mm} \times 2 \mathrm{~mm}$ at $5 \mathrm{~mm}$ depth).
Therefore, this design can be done in two steps:

1) 3D study which allows to choose the measurement method.

2) $2 \mathrm{D}$ parametric study in order to optimize the sensor [5].

Experimental measurements [6] show that the such designed sensor is well adapted to control that kind of structure and to detect deep flaws. Indeed, $15 \mathrm{~mm}$ deep flaws in aluminum can now be detected.

\section{CONCLUSION}

The $3 \mathrm{D}$ study is necessary to understand eddy currents distribution near the flaw and the influence of this perturbation on sensor response (flux density variation) at the surface of riveted assemblies. But 3D calculations are actually too expensive when $3 \mathrm{D}$ parametric study is necessary to design a sensor. So, the $2 \mathrm{D}$ simulation with an equivalent axisymmetric flaw is used to design and optimize a sensor to detect deep flaws since results are smaller but in the same way than those obtain with 3D calculation. Experimental measurements confirm that this sensor is well adapted for control of riveted assemblies in aircraft.

\section{REFERENCES}

[1] C.J. Carpenter, "Comparison of alternative formulations of 3 dimensional magnetic field and eddy currents problems at power frequencies", Proc. IEE, vol 124, pp. 1026-1034, 1977.

[2] O. Biro and $\mathrm{K}$. Preis, "On the use of magnetic vector potential in the finite element analysis of three dimensional eddy currents", IEEE transactions on Magnetics, vol 25, n 4, pp. 3145-3159, July 1989.

[3] Y. Maréchal and G. Meunier, "A new 3D AV- $\Phi-\Phi$ r formulation", IEEE Transactions on Magnetics, vol 28, $n^{\circ}$ 2, March 1992.

[4] F. Thollon and N. Burais, "Geometrical optimization of sensors for eddy currents Non Destructive Testing and Evaluation", IEEE Transactions on Magnetics, Vol 31 n $^{\circ} 3$ May 1995, pp 2026-2031..

[5] F. Thollon, B. Lebrun, N. Burais, and $Y$. Jayet, "Numerical and experimental study of eddy currents in NDT of structures and deep cracks", NDT\&E International, Vol $28 n^{\circ} 2$, pp 97-102, 1995;.

[6] F. Thollon, "Conception et optimisation de capteurs à courants de Foucault pour la détection de défauts profonds dans des matériaux amagnétiques", Ph D. Thesis Ecole Centrale de Lyon, France, 1995 